Classification

Physics Abstracts

$07.80-82.80$

\title{
Design and first applications of a post-column imaging filter
}

\author{
Ondrej L. Krivanek $\left({ }^{1}\right)$, Alexander J. Gubbens $\left({ }^{1}\right)$, Niklas Dellby $\left({ }^{1, *}\right)$ \\ and Christopher E. Meyer $\left({ }^{1}\right)$ \\ (1) Gatan Research and Development, 6678 Owens Drive, Pleasanton, CA 94588, U.S.A. \\ (Received June 15, 1992; accepted June 22, 1992)
}

\begin{abstract}
We have designed and built an imaging filter which can be attached to most standard TEMs, and is capable of operating at primary energies of up to $400 \mathrm{keV}$. The filter uses a $90^{\circ}$ magnetic sector prism, a piezoelectrically controlled energy-selecting slit, and 6 quadrupole and 5 sextupole lenses. It fully corrects second-order aberrations and distortions in images formed with electrons of selected energies, and it also produces second-order aberration-corrected spectra of variable dispersion. We show the first applications of the filter at $200 \mathrm{keV}$ primary energy, which indicate that the filter will excel in chemical mapping and spectroscopy, in improving contrast of electron images and diffraction patterns, and in making high resolution electron microscopy and diffraction more quantitative. We conclude the paper with a discussion about atomic resolution image formation using inelastically scattered electrons, and the relationship between energy-filtered diffraction patterns and images formed with electrons of the same energy.
\end{abstract}

\section{Introduction.}

Electrons traversing a thin sample in a transmission electron microscope (TEM) can be scattered both elastically and inelastically. The two types of scattering are due to different physical processes, and they result in completely different contrast in images and diffraction patterns. Forming images only with electrons of selected energies allows one to separate the different contrasts. The resultant energy-filtered images (and diffraction patterns) can provide much interesting new information about the sample, such as the distribution of different elements (chemical mapping), chemical bonding (chemical shifts), specimen thickness, etc. It also allows the acquisition of images (and diffractions patterns) formed only with elastically scattered electrons, which are highly suitable for quantitative comparison with theoretical predictions.

Two fundamentally different approaches to the formation of the energy-filtered images in a TEM are possible. In the scanning approach originating from the work of Hillier and Baker [1] and developed by Crewe and co-workers [2], the sample is illuminated by a small probe, and an energy-loss spectrum is formed using a relatively simple spectrometer. The probe is scanned across the sample, and an energy-filtered image is obtained by capturing the variation of the spectrum intensity as a function of the probe position on the specimen. In the fixed-beam approach

$\left({ }^{*}\right)$ now at laboratory of Nuclear Physics, MIT, Cambridge, MA 02139, U.S.A. 
developped by Castaing and co-workers [3, 4], a broad area of the sample is illuminated, an energyfiltered images are formed entirely electron-optically. This requires a more complicated type of spectrometers, which are called imaging filters.

The imaging filters always consist of two distinct stages. In stage 1 of the apparatus, an energyloss spectrum is formed, and electrons of specific energies are selected using an energy-selecting slit. In stage 2, the selected energy interval of the spectrum is transformed back into an image. The whole process is analogous to dark field TEM imaging, in which an electron image is transformed into a diffraction pattern in the back-focal plane of the objective lens, a part of the pattern is selected with an aperture, and then transformed back into an image. In both energy-filtered and dark field imaging, use is made of the fact that the image information is not lost in the spectrum (or diffraction) plane. The information is encoded in the momentum space, and all that one has to do to get an image back is to allow the electron beam to propagate some distance from the spectral (diffraction) plane.

The scanning and fixed-beam approaches have different strengths and weaknesses. The chief advantage of the scanning approach is that the imaging is done by manipulating the incident electron beam, which means that many different signals can be used to form the image, including elastic (high and low angle) scattering, Auger and secondary electrons, and X-rays, as well as the inelastically scattered electrons. If one uses a parallel detection spectrometer to detect a whole electron energy-loss spectrum at the same time, as many inelastic images can be acquired simultaneously as there are channels of the parallel detector (typically 1024). All the different images are acquired with just one pass of the incident beam, which makes the scanning approach very dose-efficient. On the other hand, the fact that the images are built up pixel-by-pixel makes the scanning approach rather slow, and acquisition times for energy-filtered data sets consisting of many different energies and covering many pixels typically run into several tens of minutes or even several hours.

In the fixed-beam approach, entire images are formed and detected in parallel, which makes it possible to acquire energy-filtered images consisting of $10^{6}$ and more pixels in a fraction of a second. However, only one energy can be imaged at a time, which means that a separate exposure is needed for each energy to be explored. Further, the approach is not compatible with simultaneous acquisition of other types of data such as X-ray or Auger electron images. Nevertheless, the huge speed advantage that the fixed-beam approach offers over the scanning method has made it very useful.

Imaging filters giving high quality energy-filtered images were first developed in Prof. Castaing's laboratory, which makes it a special honor for this paper to be a part of a journal issue dedicated to Prof. Castaing. The first filter originating from his laboratory is now known as the Castaing-Henry filter [3]. It uses a prism-mirror-prism combination which deflects the electron beam by $90^{\circ}$ in a magnetic prism, reflects it from an electrostatic mirror, and then deflects it by $90^{\circ}$ again in a magnetic prism. As a result, the electron beam ends up traveling parallel to the initial direction. This makes the filter highly suitable for incorporation in the optical column of a transmission electron microscope.

The electrostatic mirror prevents the Castaing-Henry filter from operating at primary energies higher than about $100 \mathrm{keV}$. Castaing and coworkers realized this early on, and developed an imaging filter using only magnetic elements [4]. The design was later taken up and improved by others [5-8], and became known as the $\Omega$-filter instead of the "Castaing-Senoussi filter", which would have been an entirely appropriate name. An $\Omega$-filter typically consists of 4 magnetic prisms which bend the electron trajectory so that it resembles the Greek letter $\Omega$ lying on its side, and is therefore also very suitable for incorporation in the column of an electron microscope.

The fact that both the Castaing-Henry and Castaing-Senoussi $(\Omega)$ filters are designed for incorporation in the microscope column has meant that they are typically not available as retrofits to 
existing electron microscopes. This has limited their popularity compared to a Hillier and Bakertype single sector spectrometer, which can be attached at the end of the electron optical column of most existing electron microscopes without needing to rebuild the microscope in any way. In this paper we report on a post-column imaging filter that combines the adaptability of the single sector spectrometer with the image-forming abilities of even the best $\Omega$-filters, and thus promises to make energy-filtered imaging, first pioneered in Prof. Castaing's laboratory, widely available.

\section{Filter design.}

Any single sector magnetic spectrometer is able to form an energy-filtered image. If one were to look closely at the scintillator that is typically situated some distance behind an energy-selecting slit in a serial-detection spectrometer (e.g. [9]), one would find a miniature version of whatever image (or diffraction pattern) was admitted into the spectrometer by its entrance aperture. Such an image, however, would contain large distortions and aberrations, arising because the cylindrical symmetry of the imaging system was lost when the electron beam traversed the magnetic sector. This was found experimentally for instance by Ajika et al., who placed a TV camera at the location of the scintillator in a single magnetic sector spectrometer [10]. They were able to record energyfiltered lattice images of silicon, but the simple electron optics of their system meant that their images had a different magnification in the $X$ than in the $Y$ direction (first-order geometric distortion), and became blurred if a larger energy interval was used (first-order energy aberration), which essentially prevented further useful work.

The first-order aberrations and distortions can be removed by placing several round lenses plus quadrupole lenses [11], or quadrupoles alone [12] downstream from the energy-selecting slit, and using the extra elements to improve the quality of the images. Our own experience with this type of approach showed us that images corrected to first-order only are acceptable as long as they are restricted to about $200 \times 200$ pixels in size. However, we also found that in larger images, second-order aberrations and distortions become highly noticeable, and, in the case of aberrations involving the electron energy, cause an irretrievable loss of image resolution. Accordingly, we investigated the possibility of removing these distortions and aberrations with sextupole lenses located at key positions between quadrupole lenses, with the whole system placed downstream from the energy-selecting slit. This investigation showed that with 4 quadrupole lenses changing the first-order properties of the electron beam (basically the energy dispersion and the vertical and horizontal beam widths), and 6 sextupoles placed between the quadrupoles in locations where the first-order beam properties varied widely, all the second-order aberrations and distortions of energy-filtered images could be eliminated $[13,14]$. Image quality is then limited only by thirdorder aberrations and distortions, and recorded images of $1024 \times 1024$ pixels are indistinguishable from images recorded by even the most highly corrected $\Omega$-filters, which employ 4 magnetic sectors and 7 sextupoles [7].

A detailed description of the electron-optical design of our filter has been given elsewhere [14]. Here we briefly review its electron optics and principal components, describe the principles governing the design of its electronics and computer software, and give several examples demonstrating the performance of the filter on practical applications.

The filter's column-mounted hardware consists of 8 principal parts (Fig. 1) :

1) Entrance aperture allowing different aperture sizes to be selected. One of the apertures is a test mask consisting of 9 holes arranged on a square grid, and is used solely for adjusting the optical properties of the filter.

2) Pre-prism weak focusing and alignment coils consisting of two dipoles, two quadrupoles, and two sextupoles. 


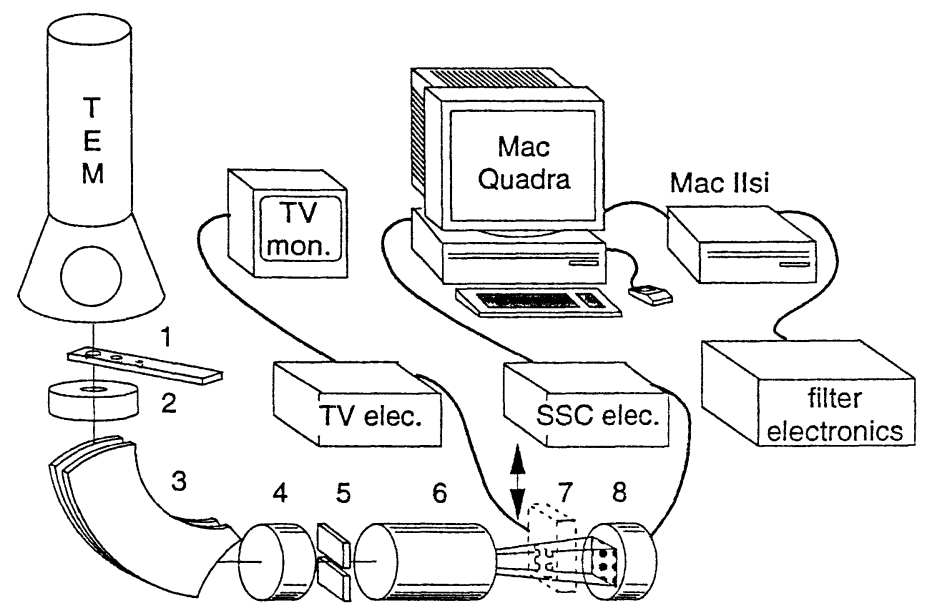

Fig. 1. - Schematic diagram showing the principal components of the imaging filter.

3) Magnetic prism of $10 \mathrm{~cm}$ bending radius and $90^{\circ}$ bending angle. The prism has curved edges and produces spectra free of all second-order aberrations. The electron beam travels through the prism inside an electrically isolated drift tube, which is used for calibrating the energy-loss spectra by applying a precise voltage to the drift tube and observing the resultant spectrum shift.

4) Two spectrum-magnifying quadrupoles, which produce a magnified, doubly-focused spectrum at the energy-selecting slit. The quadrupoles magnify the spectrum produced by the magnetic prism by about $6 \times$. This decreases the mechanical precision required of the energy-selecting slit, which would otherwise be too exacting, particularly at a primary energy of $400 \mathrm{keV}$, at which the dispersion of the unaided prism is only $0.5 \mu \mathrm{m}$ per $\mathrm{eV}$.

5) Energy-selecting slit, whose width can be changed by applying a suitable voltage to a piezoelectric element. The slit allows an energy interval to be selected for energy-filtered imaging, and it can be pneumatically retracted for observing wide-range energy-loss spectra.

6) A quadrupole-sextupole imaging assembly consisting of 4 quadrupoles, 5 sextupoles, and a number of alignment coils. The quadrupoles and sextupoles can either produce energy-filtered images, or their excitations can be changed so that they produce spectra of adjustable width and variable dispersion. (A sixth sextupole that was present in our original design was left out because it was found that one of the second-order aberrations was so small that it did not need correcting).

7) Pneumatically retractable TV-rate CCD camera which allows energy-filtered images or spectra to be observed live, and is used for adjusting the filter's optics and in searching for interesting specimen areas.

8) Slow-scan CCD (SSC) camera [15] of up to $1024 \times 1024$ pixels, which allows photographicquality images to be recorded electronically with a good DQE, a wide dynamic range, and with integration times stretching up to several minutes. The SSC images are immediately available in a computer for further processing, and the camera also permits electron energy-loss spectra to be recorded in parallel with excellent DQE and good dynamic range.

The power to all the optical elements such as the magnetic prism, quadrupoles, sextupoles, alignment coils, the electrically isolated drift tube, and the energy-selecting slit is provided by computer-controlled power supplies. The software controlling the supplies runs on a Macintosh II or Quadra, and is mouse-driven and simple to use. Another Macintosh acquires, displays, and processes images from the SSC, and controls the high voltage and several lenses of the TEM. It 
also transforms 2D images of spectra acquired by the SSC into 1D spectrum profiles, and passes them to the Gatan energy-loss spectrum-processing software (EL/P) for quantitative analysis.

By controlling the high voltage of the TEM, the user is able to focus an image using the strong elastically scattered signal, and then go to an energy-loss without having to worry whether the new image will be out of focus. This is because changing the primary energy from $E_{0}$ to $\left(E_{0}+\Delta E\right)$ while keeping the energy of the electrons selected by the slit equal to $E_{0}$ ensures that inelastically scattered electrons admitted through the slit will have traveled through the microscope imaging column in precisely the same way as the elastically scattered electrons did before the primary energy was changed. The image therefore cannot go out of focus, no matter how large the coefficient of chromatic aberration of the objective lens is, or how large the energy-loss is. By comparison, if the selection of a particular energy-loss was accomplished by changing the energy selected by the imaging filter, the chromatic aberration of the objective lens of the TEM would defocus the image. Because the intensity of the inelastically scattered images is typically quite weak (compared to elastic images), precise refocusing would then be much more difficult. The focus of the incident illumination changes slightly when the primary energy is changed, but this effect is easily compensated by a minor change of the excitation of the final condenser lens.

Because the imaging filter adds an extra magnification of about $15 \times$ relative to the image as it appeared on the viewing screen of the TEM, we reduce the magnification of the TEM imaging column by about $15 \times$ whenever the operation switches from the microscope viewing screen to the imaging filter. This is done by controlling the last two projector lenses by the filter's computer. The final projector is weakened, and the preceding projector is strengthened, in order to keep the final crossover at a constant height. The resultant image is typically only a few mm wide at the level of the viewing screen, precisely as required when there is another section of the optical column located below the viewing screen. Because the reduced size of the image makes it more susceptible to the influence of stray AC magnetic fields, it is essential that the viewing chamber (and also the camera chamber if located just below the viewing chamber) be made out of a magnetically shielding material such as soft iron rather than brass or stainless steel.

The software controlling the imaging filter and the software controlling the acquisition of the images with the slow-scan CCD camera currently run on two separate Macintoshes. The two Macs communicate over a high-speed serial line, and the user can interact with both of them via a single keyboard and monitor. The majority of the optical elements of the filter are programmed in a way that is similar to the preset programs of the imaging lenses of a TEM. The user normally only controls whether the filter is producing images or spectra, the selected energy, the spectrum dispersion, whether the energy-selecting slit is in or out, and the slit width (which is calibrated in $\mathrm{eV}$ units). The user can also touch up parameters such as the first-order and second-order focus of the spectrum, and the first-order chromatic and geometric properties of the energy-filtered images. This can all be accomplished with 4 principal and 8 secondary controls, and is quite easy even for beginning electron microscopists. Full access to the programming of the individual quadrupoles and sextupoles is reserved for Gatan installation engineers.

\section{Operation of the filter.}

The imaging filter has two modes of operation: image mode and spectrum mode. In image mode, the entrance aperture of the filter selects a part of the image (or diffraction pattern) that is projected by the microscope into the viewing chamber, and the filter produces an energy-filtered version of the image either on the TV-rate camera or, if the TV camera is retracted, on the slow-scan CCD (SSC) camera. In spectrum mode, a focused spectrum is formed at the final detector, and the spectrum's dispersion can be varied as needed, much like with the Gatan parallel-detection 
electron energy-loss spectrometer (PEELS) [16]. The slit is typically withdrawn in the spectrum mode. Before switching to imaging, the slit is inserted, and the appropriate energy interval is selected while observing the spectrum. Alternately, the slit can be left out for an unfiltered image.

Spectra produced at the energy-selecting slit or at the SSC are free of all first and secondorder aberrations, and images formed by the imaging assembly are also free of all important first and second-order aberrations and distortions [14]. Because the filter focuses on the final TEM crossover which occurs just below the last projector lens under all normal TEM imaging and diffraction conditions, operation of the filter and of the TEM are largely independent. The filter simply produces an energy-filtered version of whatever image or diffraction pattern was in the center of the microscope viewing screen just before the screen was lifted up, and it does not degrade the attainable resolution. The operation of the filter is therefore very simple. Indeed, it is our experience that the casual observer, when looking at the image on the filter's TV monitor while someone else is using the filter, is not even aware that the electron beam traversed an energy-selecting slit as well as all the optical elements of the filter, at least not until the operator stops looking at elastic images, and starts exploring the various inelastic scattering mechanisms.

\section{Applications of the filter.}

The total time that has so far been available for testing our filter on actual specimens is still measured in days rather than months or years. However, our initial experience with the filter indicates that it can perform all the types of experiments that have been done with imaging filters so far [e.g., 17], and that its ability to work with most existing TEMs will give it unique capabilities not available with imaging filters incorporated into the imaging column of just one type of a TEM. Accordingly, we first demonstrate the performance of the filter with a series of images of ferritin, which is a standard TEM test specimen sused for demonstrating the ability to do chemical mapping. We then show energy-filtered high resolution images of thorium oxide, and discuss the origin of the high resolution contrast in these images.

Figure 2 shows ferritin particles imaged at $200 \mathrm{keV}$ with the imaging filter attached to a JEOL 2010 TEM using an $\mathrm{LaB}_{6}$ gun. There are several particles in the field of view. The elastic-only image (a) shows them as dark, they are not visible in the image taken with the pre-Fe $\mathbf{M}_{2,3}$ edge electrons, and they show as bright in images taken with electrons just above the iron $\mathbf{M}_{2,3}$ edge at $54 \mathrm{eV}$ (c) and the $\mathrm{L}_{2,3}$ edge at $708 \mathrm{eV}$ (d). The spatial resolution is of the order of $3 \mathrm{~nm}$, and appears to be about the same in the $M_{2,3}$ image (c) as in the rather noisy $L_{2,3}$ image (d), which was recorded with the same illumination conditions, and $8 \times$ the acquisition time of image (c). This demonstrates that similar to the recent trend in parallel-detection EELS to use lowerenergy edges [18], chemical mapping by energy-filtered imaging is often better performed with low energy-edges than with high energy ones. Accurate quantification of the low-loss images may require that as many as 10 or 20 images be recorded using narrow energy intervals, and that one employs the same least squares spectrum-fitting and difference techniques that have been shown to be optimal for parallel-detection EELS $[18,19]$. This will increase the electron dose by an order of magnitude beyond the PEELS dose, but it will make it possible to acquire quantitative chemical maps as large as $1024 \times 1024$ pixel in a few tens of second. The required software will incorporate elements of the spectrum-image approach originally developed for PEELS mapping [20,21], and is now being written in our laboratory.

Figure 3 shows an energy-filtered (elastic scattering only) high resolution image of thorium pyromellitate deposited on graphite and imaged at $200 \mathrm{keV}$ with the filter attached to a JEOL 2010 TEM. The pyromellitate reduces under the electron beam to thorium oxide, and the recorded structure image is due to a particle of the oxide. The appearance of the image is similar to what 

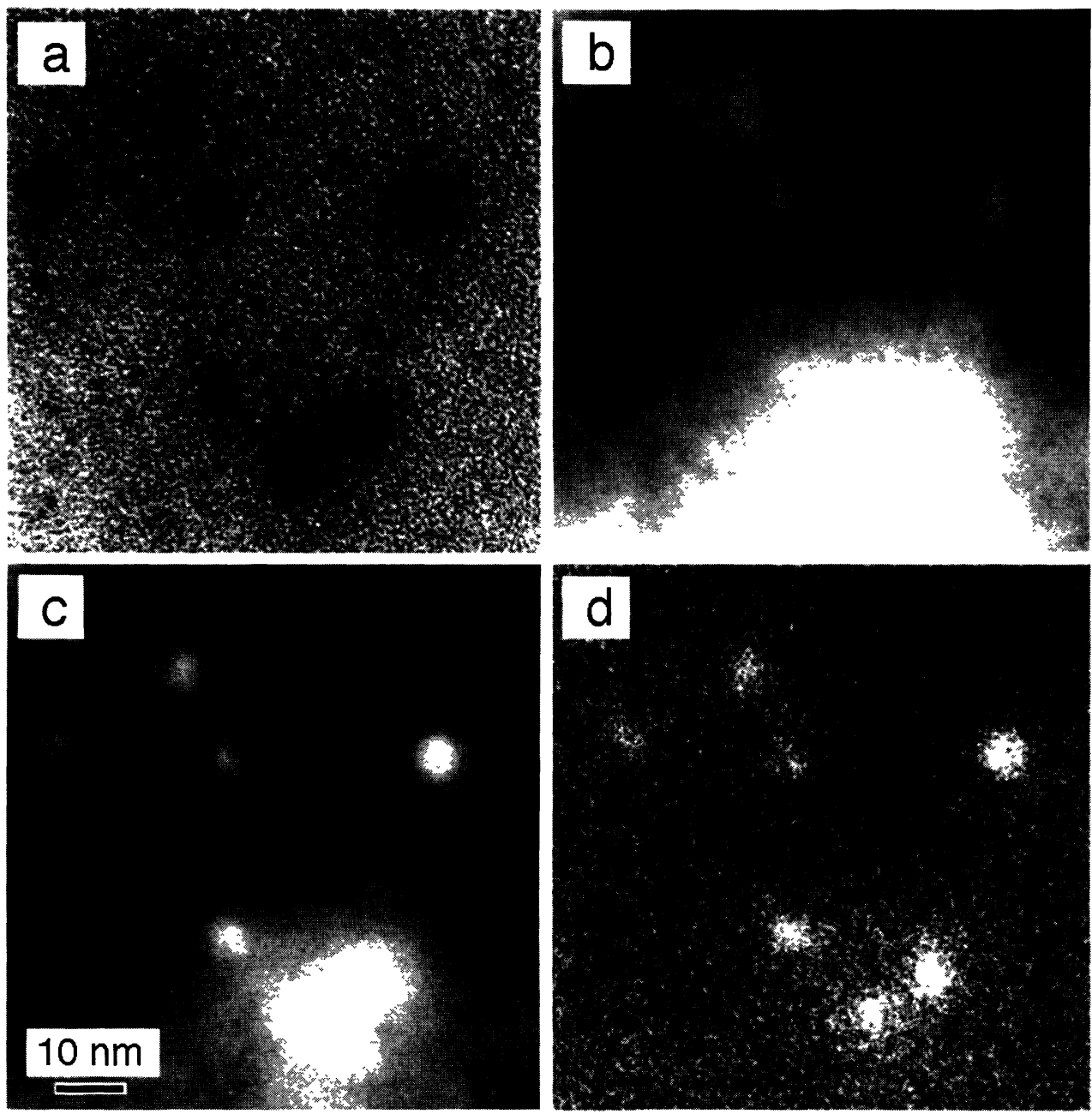

Fig. 2. - Images of ferritin particles on amorphous carbon : a) no loss ( 0.5 acquisition time, $20 \mathrm{eV}$ wide slit), b) 30-50 eV losses ( $2 \mathrm{~s})$, c) 50-70 eV losses ( $2 \mathrm{~s}$ ) and d) 700-750 eV losses (16 s).

could have been obtained by the unaided microscope. However, because the image is energyfiltered, its contrast is not diminished by the presence of inelastic scattering. This makes the image clearer than the equivalent unfiltered image. Even more importantly, since there is no inelastic contribution, absolute comparison of the image contrast with the predictions of multislice or Bloch wave image calculations should be readily possible. By making such precise experiment-theory matching practical, the imaging filter promises to elevate high resolution electron microscopy to a higher level of absolute quantification, and to a more accurate understanding of all the contrast mechanisms involved.

Figure 4 shows an energy-loss spectrum of a thorium pyromellitate particle recorded with the imaging filter operating in the spectrum mode. The same spectrum is first shown as a twodimensional image that was recorded by the slow-scan CCD camera (a), and secondly as a "fat" line profile (a line profile which integrates in a direction perpendicular to the profile direction) 


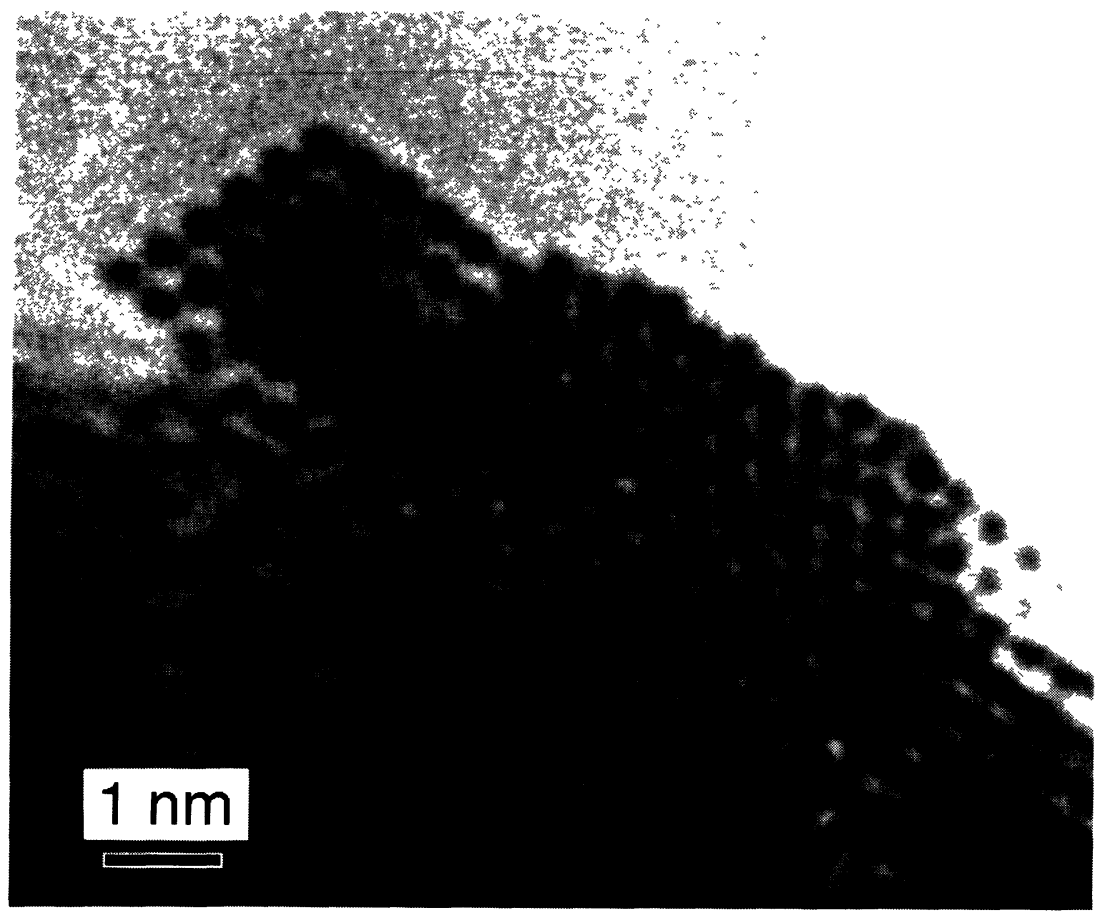

Fig. 3. - Elastic-only energy-filtered image of a thorium oxide particle. JEOL 2010, $200 \mathrm{keV}$, acquisition time 0.5 seconds. Specimen courtesy Prof. Hashimoto.

of the spectrum (b). The profile clearly shows the thorium $\mathrm{O}_{4,5}$ edge, and demonstrates that the filter gives similar quality spectra as a parallel-detection spectrometer.

Figure 5 shows images of a group of thorium oxide particles taken using an energy-selecting slit about $10 \mathrm{eV}$ wide, and selecting either elastically scattered electrons (a), or electrons with energies just below (b) and just above (c) the thorium $\mathrm{O}_{4,5}$ edge. The energy intervals used for (b) and (c) are indicated in figure $4 \mathrm{~b}$. The elastic-only image (a) again shows the details of the atomic structure of the sample, even though it is less clear than figure 3, probably because the specimen orientation was further away from a major zone axis. The image structure largely disappears in the pre-edge image (b). It reappears in the post-edge image (c), but with reduced contrast. The fact that high resolution contrast appears in the image formed largely by electrons that have ionized the thorium $\mathrm{O}_{4,5}$ shell indicates that the image may be a chemical map showing the location of the thorium atoms, and that the goal of chemical identification with atomic resolution may thus be within reach. However, before making such a conclusion, we need to examine whether there are other possible mechansims that could give rise to the observed contrast.

\section{Origin of high resolution contrast in energy-filtered images.}

Before deciding that the contrast in image $5 \mathrm{c}$ is entirely due to inelastically scattered electrons, one must consider that elastic scattering takes place at the same time as inelastic scattering, and can contribute to the contrast in the inelastic images. Such a contribution involves mixed inelasticelastic scattering, in which the inelastic scattering alters the electron energy, and at the same time 
a
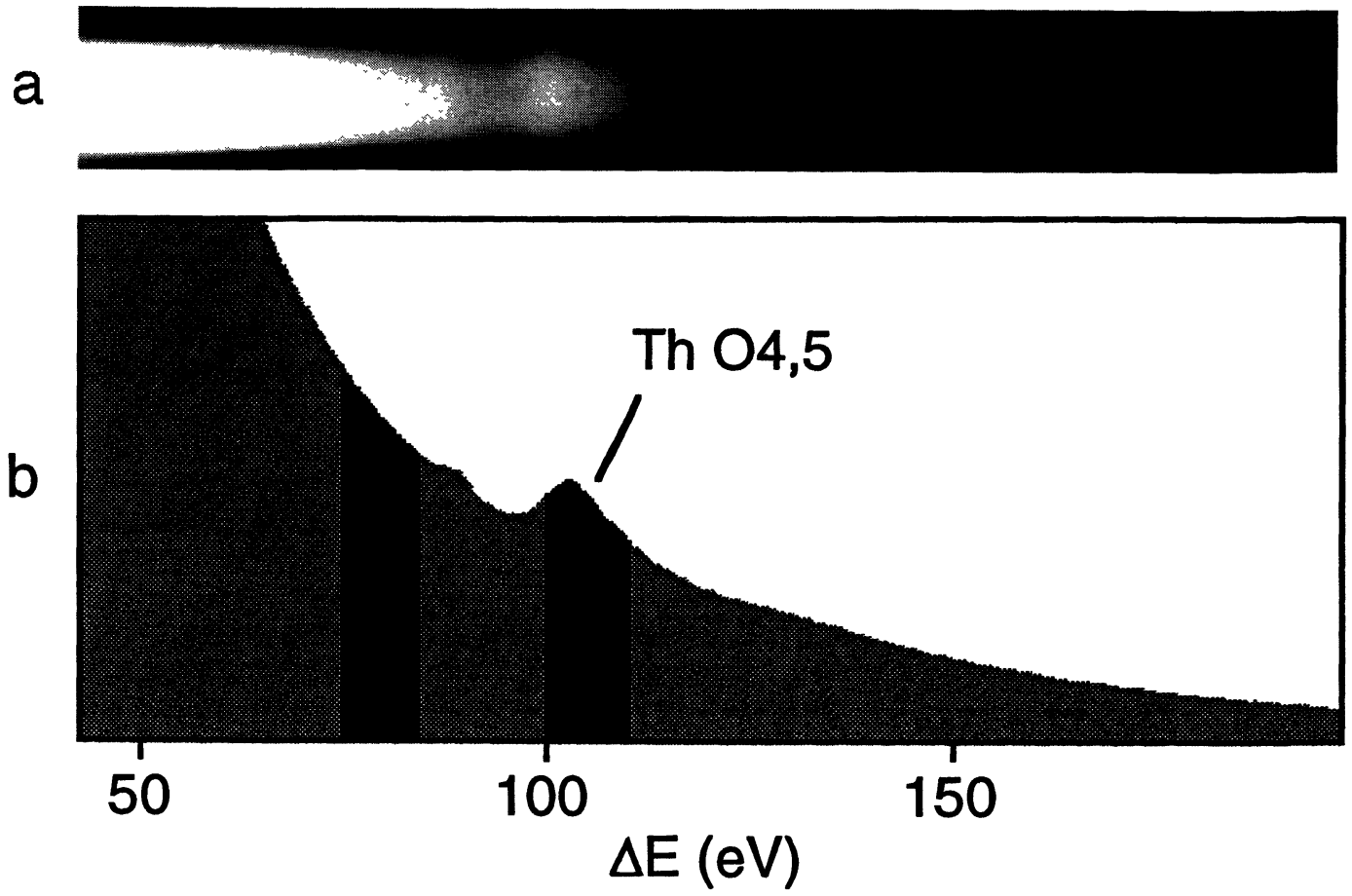

Fig. 4. - Energy-loss spectrum of a thorium oxide particle : a) as recorded by the filter's slow-scan CCD camera ; b) A line profile obtained by integrating perpendicular to the dispersion direction across the whole width of the spectrum. Acquisition time 1.6 seconds.
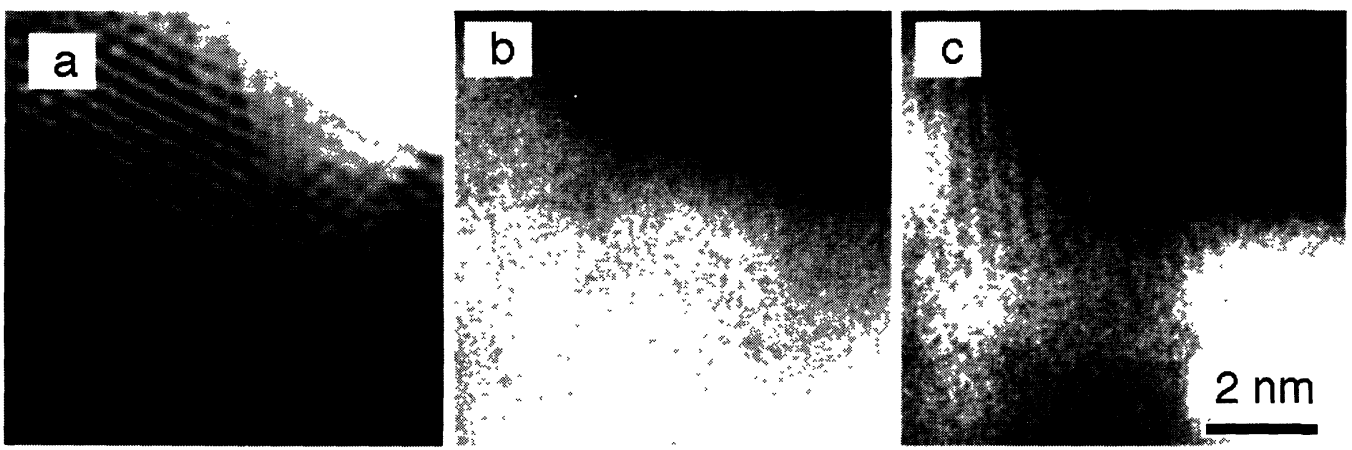

Fig. 5. - Energy-filtered images of a group of thorium oxide particles : a) elastic, b) $80 \mathrm{eV}$ energy-loss (pre-Th $\left.\mathrm{O}_{4,5}\right), 100 \mathrm{eV}$ loss (post-Th $\mathrm{O}_{4,5}$ ). Slit width about $10 \mathrm{eV}$, acquisition times $0.5 \mathrm{~s} \mathrm{(a)} \mathrm{and} 8 \mathrm{~s}$ (b and c).

preserves the elastic contrast. The appearance of elastic scattering contrast in inelastic energyfiltered images is well known from both STEM and TEM work [10,12, 22, 23], and is the primary mechanism for image contrast in low-loss (plasmon) energy-filtered images.

Probably the best way to determine the origin of the contrast in inelastic images is to examine 
the diffraction patterns formed by electrons of the various energies used for the imaging. Such patterns typically reveal the physics behind the contrast of the images, and they bypass the complicating influence of the imaging properties of the microscope. Figure 6 shows schematically the expected behavior on a hypothetical sample consisting of an artificial superlattice formed by two elements whose atomic numbers are similar, so that their elastic scattering is nearly the same. The elastic energy-filtered diffraction pattern (a) will contain a row of strong spots due to the basic lattice, plus weak reflections due to the superlattice. The corresponding high resolution image (a') will show the basic lattice strongly, with an additional weak modulation due to the superlattice.
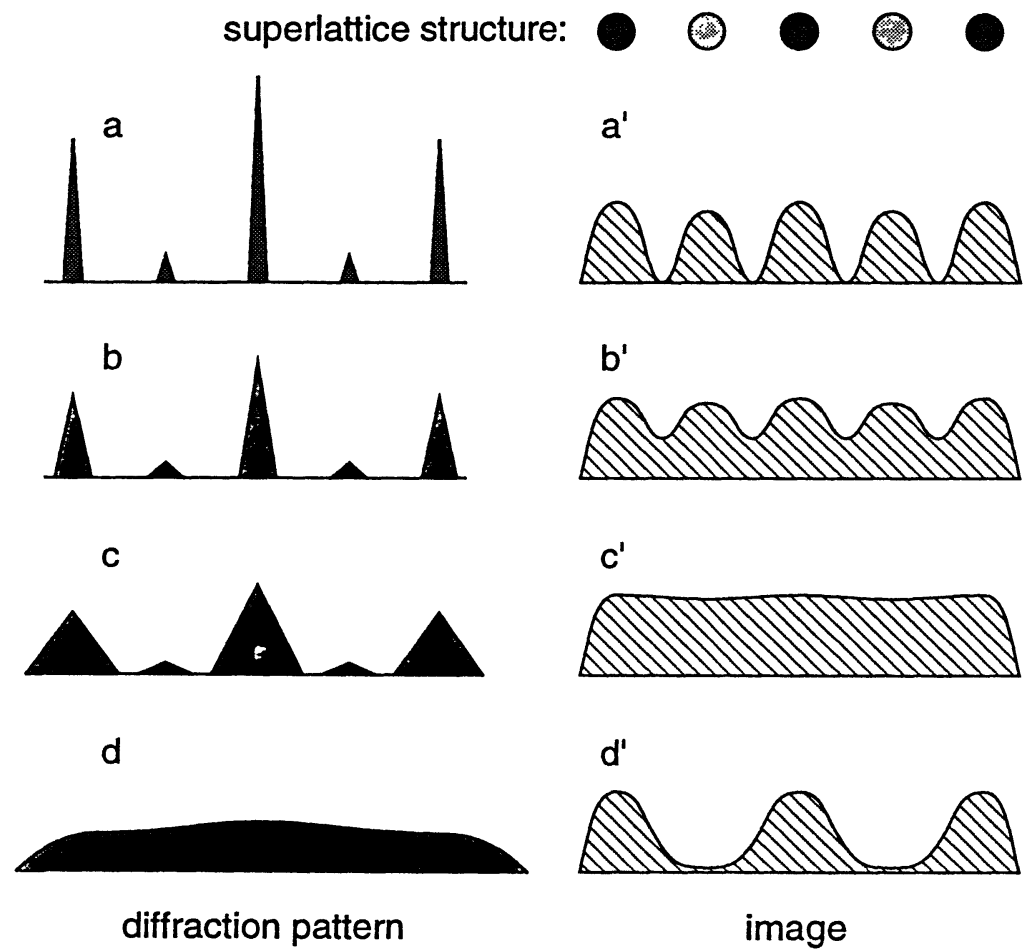

Fig. 6. - Schematic representation of the energy-loss dependence of a diffraction pattern and the resultant high resolution image of an artificial superlattice consisting of two elements with similar atomic numbers : $\left.a, a^{\prime}\right)$ elastic diffraction pattern and image, b, b') diffraction pattern and image formed with electrons that have lost a small amount of energy, $c, c^{\prime}$ ) diffraction pattern and image formed with electrons that have lost an intermediate amount of energy, and d, d') diffraction pattern and image formed with electrons that have lost a high amount of energy.

At an energy-loss of several tens of $\mathrm{eV}$ (for instance, at the plasmon energy), the Bragg spots will be broadened by the additional angular spread due to the inelastic scattering (b). The width of the additional spread will typically be equal to two times the characteristic angle for inelastic scattering $\theta_{E}$, given by $\theta_{E}=\Delta E / 2 E_{0}$, where $\Delta E$ is the energy-loss, and $E_{0}$ is the primary electron energy. At $E_{0}=200 \mathrm{keV}$ and $\Delta E=20 \mathrm{eV}, \theta_{E}=0.05 \mathrm{mrad}$, which is about two orders of magnitude smaller than the typical Bragg angle. Because such low-angle inelastic scattering hardly changes the intensity distribution of the elastic diffraction pattern, the corresponding inelastic image (b') strongly resembles the elastic one. The most significant difference is that the inelastic image is 
given by a product of the inelastic intensity times the elastic image contrast, i.e., the image is more intense in sample regions that give stronger inelastic scattering. Another difference is that in normal TEM imaging, the effective defocus of the inelastic image contribution is different from the elastic defocus, because of the chromatic aberration of the microscope's objective lens. In energy-filtered TEM in which different energies are selected by changing the microscope primary energy (as here) as well as in STEM, however, the defocus of the inelastic image is the same as for the elastic image. A further difference is that some image contrast is lost in the inelastic image, because the inelastic scattering decreases the coherence of the incident beam.

At higher energy-losses, the broadening of the Bragg spots increases further (c), and the resultant loss of beam coherence increases so much that the image contrast due to elastic scattering is effectively destroyed ( $\left.c^{\prime}\right)$. Even at such energy-losses, however, the width of the inelastic scattering distribution is likely to be less than the separation between the weak Bragg reflections due to the superlattice, which means that the inelastic scattering is not sufficiently localized to allow the superlattice to be resolved purely by inelastic scattering, and the image is simply featureless.

At still higher energy-losses, the inelastic scattering will broaden the Bragg reflections so much that they will no longer be distinguishable in the diffraction pattern (d). It is at this energy-loss that the inelastic scattering will finally become sufficiently localized so that inelastic images of the individual atoms will become narrow enough to enable the superlattice to be directly resolved (d'). If one of the two elements has an ionization edge at this energy value, it will then only be a matter of signal strength whether the superlattice will indeed become resolvable in the inelastic image. Note that because the inelastic scattering is incoherent (an electron scatters inelastically only from one particular atom rather than from a whole set of crystal planes as in elastic scattering), the diffraction pattern corresponding to the high resolution inelastic image will not show any distinct periodicities - it will simply be blurry, and no individual Bragg spots will be distinguishable.

Figure 7 shows a series of diffraction patterns of the thorium oxide sample recorded at increasing energy-losses. The elastic-only pattern (a) shows well defined Bragg spots originating from the supporting graphite film, as well as Bragg spots due to the oxide. The $26 \mathrm{eV}$ loss pattern (b) shows the scattering distribution of plasmon-loss electrons. It strongly resembles the elastic-only distribution, with the main difference being that the Bragg spots are slightly more diffuse. This is because the plasmon scattering is sharply forward-peaked, as discussed above, and it means that any lattice fringes seen in a plasmon-loss image of the sample are actually due to elastic scattering. The $100 \mathrm{eV}$ energy-loss pattern (c) also strongly resembles the elastic pattern. This means that any high resolution contrast in the $100 \mathrm{eV}$ loss image of the sample is due to the elastic scattering, and that such an image is therefore not a chemical map. This is probably the explanation for the contrast seen in the thorium $\mathrm{O}_{4,5}$ image $(5 \mathrm{c})$. The reason that similar contrast was not observed as clearly in the pre-edge image (5b) is probably that the total intensity in the pre-edge image was weaker, and that the pre-edge inelastic scattering distribution was actually wider, thereby attenuating the elastic contrast more strongly. (The larger angular width of pre-edge inelastic scattering arises because the scattering becomes narrower when a strong new inner-shell loss edge appears in the spectrum.) The $300 \mathrm{eV}$ loss diffraction pattern (d) finally shows large blurring, indicating that the inelastic scattering distribution may be sufficiently broad for atomic structure to become directly resolvable in inelastic images. However, images of the thorium oxide recorded above the thorium $\mathrm{N}_{6,7}$ ionization energy (maximum at about $450 \mathrm{eV}$ ) and the oxygen $\mathrm{K}$-shell energy $(530 \mathrm{eV})$ showed no atomic-level contrast. This was probably because these images were quite weak in intensity, necessitating recording times of 20 seconds and longer, which made it easy for instrumental factors such as specimen drift to destroy any high resolution image details.

In summary, high resolution inelastic images showing atomic-level distribution of different chemical species should be obtainable in principle, but one needs to be careful not to confuse them with images showing contrast due to combined elastic-inelastic scattering. Atomic-resolution 

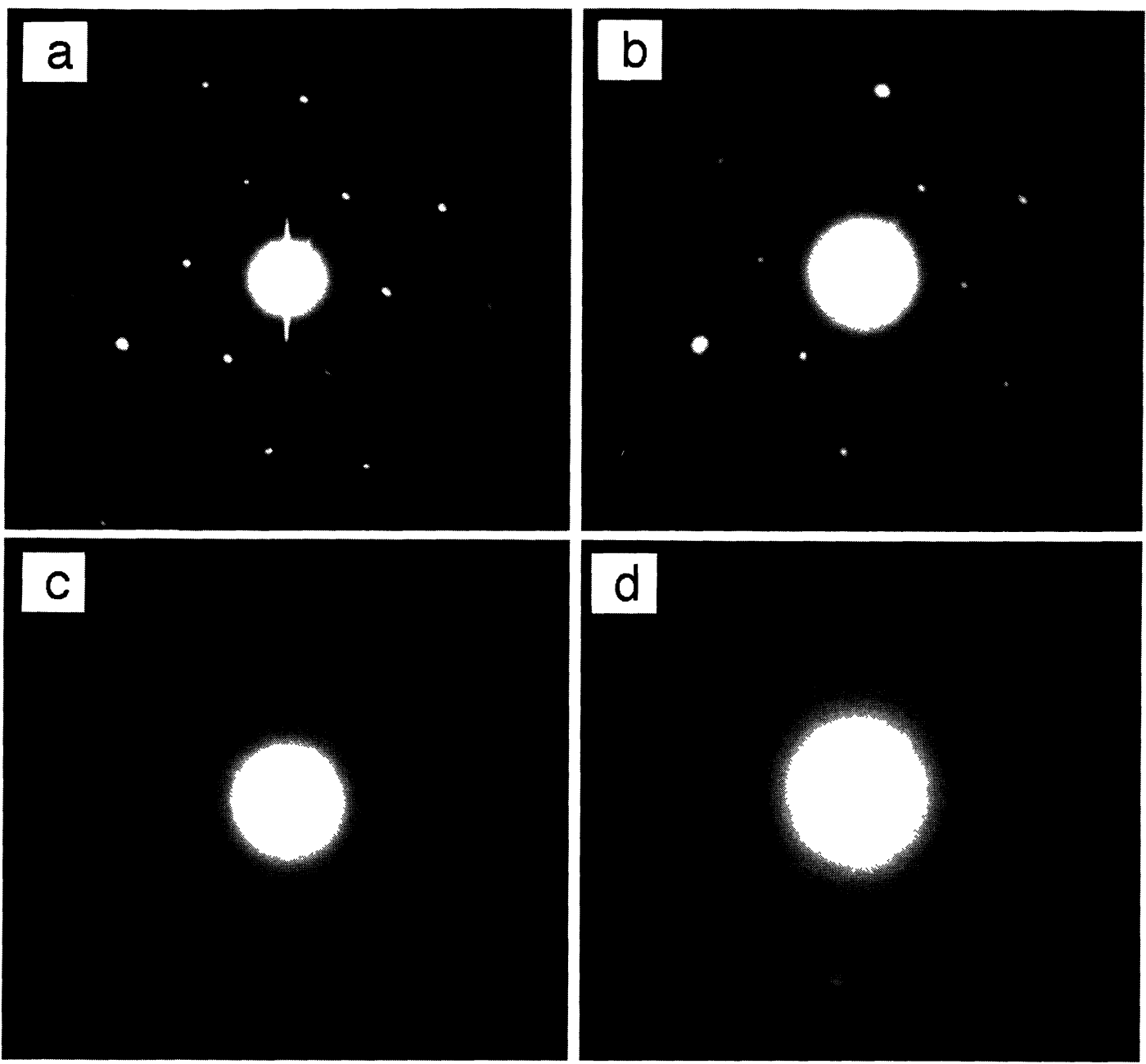

Fig. 7. - Energy-filtered diffraction patterns of thorium oxide particles lying on the graphote supporting film : a) elastic, b) $26 \mathrm{eV}$ energy-loss, c) $100 \mathrm{eV}$ energy-loss, d) $300 \mathrm{eV}$ energy-loss. Slit width about $10 \mathrm{eV}$, acquisition times $0.8,0.8,1.6$ and 3.2 seconds respectively.

chemical images should be obtainable primarily with higher energy-loss edges, but possibly also with lower energy edges, provided that one images with electrons whose energies are some distance above the edge, so that their angular distribution becomes broadened due to the appearance of the Bethe ridge [24]. In either case, the intensity of the images will be quite low, and instrumental factors such as specimen drift will easily wipe out the image features. Examining the diffraction pattern formed by electrons of the same energy-loss as used for the imaging will help clarify whether the inelastic images can show atomic-resolution chemical contrast or not.

\section{Conclusion.}

The electron-optical performance of our new post-column imaging filter matches the performance of even the best in-column filters. Our instrument can be attached to most TEMs, provides distor- 
tion and aberration-free energy-filtered images and spectra of any sample, and makes the results immediately available in a computer for processing and analysis. Fully computerized electronics and user-friendly software make the instrument simple to operate, without compromising its power.

The field of inelastic imaging is a large one, and the full exploration of the possibilities openend up by the filter is likely to take some time. First experiments with the filter indicate that it will acquire chemical maps with a resolution of a few $\mathrm{nm}$ in seconds, and that it will provide images and diffraction patterns of increased contrast and improved interpretability. Atomic-resolution chemcial maps may also be obtainable with the filter, but their feasibility still remains to be convincingly demonstrated.

\section{Acknowledgements.}

We are grateful to Prof. H. Hashimoto of Okayama University for his interest in the filter and for providing the thorium pyromellitate sample, and to Peter Swann for continuous encouragement.

\section{References}

[1] Hillier J. and BAKer R.F., J. Appl. Phys. 15 (1944) 663.

[2] CREWE A.V., ISAACSON M. and JOHNSON D., Rev. Sci Instrum. 42 (1971) 411.

[3] Castaing R. and Henry L., C.R. Acad. Sci. Paris B255 (1962) 76.

[4] SENOUSSI S., HENRY L. and CASTAING R., J. Microsc. 11 (1971) 19.

[5] ZANCHI G., PEREZ J.-P. and SEVEly J., Optik 43 (1975) 495.

[6] KRAhl D., HeRmann K.H. and Kunath W., Proc. 9th Int. EM Congress, J. M. Sturgess Ed. (Microscopical Society of Canada, Toronto, 1978), vol 1, p. 42.

[7] Rose H. and PEJAS W., Optik 54 (1979) 235.

[8] LANIO S., Optik 73 (1986) 99.

[9] KRIVANEK O.L. and SWANN P.R., in: Quantitative Microanalysis with High Spatial Resolution, G.W. Lorimer, M.H. Jacobs and P. Doig Eds. (The Metals Society, London, 1981) p. 136.

[10] AJIKa N., HaShimoto H., YamaguCHI K. and ENDOH H., Jpn J. Appl. Phys. 24 (1985) L41.

[11] Shuman H. and SomlYo A.P., Proc. Nat. Acad. Sci USA 79 (1982) 106.

[12] KRIVANEK O.L. and AHN C.C., Electron Microscopy 1986, T. Imura, S. Maruse and T. Suzuki Eds. (Jap. Soc. Electron Microscopy, Tokyo, Japan, 1986) vol. 1, p. 519.

[13] KRIVANEK O.L., US patent \# 4,851,670.

[14] KRIVANEK O.L., GubBENS A.J. and DellbY N., Microsc. Microanal. Microstruct. 2 (1991) 315.

[15] MoONEY P.M. et al. Electron Microscopy 1990, L.D. Peachey and D.B. Williams Eds. (San Francisco Press, San Francisco, 1990) vol. 1, p. 104.

[16 KRIVANEK O.L., AHN C.C., and KEENEY R.B., Ultramicroscopy 22 (1987) 103.

[17] Proceedings of European Workshop on Electron Spectroscopic Imaging and Analysis Techniques, L. Reimer Ed., Ultramicroscopy 32 (1990) 1-89.

[18] LEAPMAN R.D. and HUNT J.A., Microsc. Microanal. Microstruct. 2 (1991) 231.

[19] ShUMAN H. and KRUIT P., Rev. Sci. Instrum. 56 (1985) 231.

[20] HUNT J.A. and WILLIAMS D.B., Ultramicroscopy 38 (1991) 47.

[21] LEAPMAN R.D. and HUNT J.A., in: Microscopy, The Key Research Tool (Electron Microscopy Society of America, Woods Hole, MA, USA, 1992) p. 39.

[22] Craven A.J. and CollieX C., in: Proc. 35-th EMSA meet, G.W. Bailey Ed. (Claitor's, Baton Rouge, Louisiana, USA, 1977) p. 242.

[23] KRIVANEK O.L., AHN C.C. and WOOD G.J., Ultramicroscopy 33 (1990) 177.

[24] EGERTON R.F., Electron Energy Loss Spectroscopy in a Transmission Electron Microscope (Plenum Press, New York, 1986). 